\title{
Family cohesion, acculturation, maternal cortisol, and preterm birth in Mexican-American women
}

This article was published in the following Dove Press journal:

International Journal of Women's Health

3 May 2013

Number of times this article has been viewed

\author{
R Jeanne Ruiz' \\ Rita H Pickler ${ }^{2}$ \\ C Nathan Marti ${ }^{3}$ \\ Nancy Jallo ${ }^{4}$ \\ 'College of Nursing, The Ohio State \\ University, Columbus, OH, USA; \\ ${ }^{2}$ Department of Patient Services, \\ Cincinnati Children's Hospital Medical \\ Center, Cincinnati, OH, USA; ${ }^{3}$ Abacist \\ Analytics, Austin, TX, USA; ${ }^{4}$ School \\ of Nursing, Virginia Commonwealth \\ University, Richmond, VA, USA
}

Correspondence: $\mathrm{R}$ Jeanne Ruiz

216 Pioneer Passage Bastrop, TX, USA

$\mathrm{Tel}+\mathrm{I} 51232 \mid 4722$

Fax+I 5123214722

Email jruiz@con.ohio-state.edu
Objective: To examine the potential moderating effects of family cohesion and acculturation on the physiological stress response (cortisol) as a predictor of preterm birth (PTB) in pregnant Mexican-American women.

Methods: The sample included 470 participants; 33 had preterm births. All participants were self-identified as Mexican-American. In this cross-sectional study, family cohesion was measured by a self-report questionnaire. Acculturation was measured by self-report questionnaire as well as by years in the United States and country of birth. Stress was measured by serum cortisol. All measures were obtained at 22-24 weeks gestation. Additional data including history of PTB were obtained from the health record. Data analysis was primarily conducted using logistic regression.

Results: The relationship between stress and PTB was predicted by family cohesion (estimate/ standard error $[\mathrm{E} / \mathrm{SE}]=-2.46, P=0.014)$ and acculturation $(\mathrm{E} / \mathrm{SE}=2.56, P=0.011)$. In addition, there was an interaction between family cohesion and history of previous PTB $(\mathrm{E} / \mathrm{SE}=-2.12$, $P=0.035)$.

Conclusion: Results indicate that the impact of cortisol on PTB is predicted by acculturation and family cohesion such that higher levels of cortisol in conjunction with higher levels of acculturation and lower levels of family cohesion are associated with increased risk of PTB. In addition, low family cohesion in combination with a history of PTB was associated with higher levels of PTB. Assessment of family cohesion, including problem solving, adherence to family decisions, family shared space, and activity, should be included as part of prenatal assessment for risk of PTB. Subsequently, interventions that focus on improving the individual's response to an imbalance in family functioning are needed. In addition, prenatal assessment of level of acculturation may also identify those who are at risk for PTB.

Keywords: preterm birth, cortisol, family cohesion, Mexican-American, stress, acculturation

\section{Introduction}

Preterm birth (PTB), birth before 37 weeks gestation, is the most pressing problem in obstetrics today. It is one of the leading causes of infant mortality and the leading cause of infant morbidity and thus has tremendous health and economic costs for the infant, family, and society. ${ }^{1}$ There is evidence to suggest that PTB is a multifactorial disease that may be the end point of subacute or chronic pathophysiologic changes that occur before clinical symptoms of preterm labor are present. ${ }^{2} \mathrm{~A}$ history of PTB is one of the strongest known risk factors for PTB; women who have delivered preterm once tend to deliver preterm in subsequent pregnancies, ${ }^{3}$ indicating that persistent characteristics of the mother influence the probability of PTB. 
PTB is likely the result of an overlapping set of interrelated social and environmental factors. ${ }^{1,2}$ One of these social factors is stress; there is much evidence that suggests that women experiencing increased psychological or social stress during pregnancy are at significantly increased risk for preterm birth. ${ }^{4-8}$ Although differing research methods make comparisons of effect size across studies problematic to interpret, it has been reported that pregnant women reporting high levels of psychosocial stress have a 25\%-60\% increased risk for PTB compared with women reporting low levels of stress. $^{4}$

The path from stress to PTB is complex, but stress biology suggests several plausible directions, including the maternal-placental-fetal neuroendocrine systems. ${ }^{9-11}$ It is postulated that, when the fetal-placental unit is exposed to high levels of stress during pregnancy, a neuroendocrine response is triggered with subsequent maternal endocrine changes and preterm birth. ${ }^{9}$ The hypothalamic-pituitaryadrenal (HPA) axis and the locus ceruleus norepinephrine (LC/NE) system are important pathways controlling the stress response. Increased glucocorticoid activity (ie, increased cortisol activity) is a characteristic of stress, having a wide range of effects in the reproductive system. ${ }^{12}$ Maternal glucocorticoids such as cortisol are considered a major candidate for the mediation of prenatal stress to the fetus and a critical endocrine mediator of birth outcomes. ${ }^{4,12,13}$ Under a state of chronic stress, the regulatory mechanisms of the stress response fail, leading to changes in cortisol levels. ${ }^{9}$ Some researchers suggest higher levels of acute stress are associated with higher levels of cortisol, a biological marker of stress, in pregnant women ${ }^{13-15}$ and that elevated maternal cortisol levels are related to preterm birth. ${ }^{16-18}$ A recent review of 15 studies examining the relationship between cortisol and PTB revealed that the majority of studies reported a relationship between maternal cortisol levels and PTB. In addition, the diurnal slope of cortisol and elevations in the first morning levels of cortisol have been shown to be linked to PTB. ${ }^{19,20}$ The bluntness of the slope of salivary cortisol (ie, a flatter response) has also been linked to chronic stress and has been shown to be important to predict adverse birth outcomes; cortisol levels may thus appear lower in a onetime sample. ${ }^{20}$ Several studies indicate that persons who have experienced chronic stress have lower baseline cortisol levels and a flattening of the diurnal cortisol slope. ${ }^{21,22}$ While stress represents one of the few established direct effects that predict PTB, it is not sufficient to reliably predict this important pregnancy outcome. Hence, a step toward increased understanding of the impact of stress on PTB is the investigation of factors that may affect stress on women during pregnancy.

One potential factor in the complex stress-preterm birth connection is ethnicity. ${ }^{23}$ The Mexican-American population is of particular interest in this developing area of science and clinical practice. The Mexican-American population has grown by $43 \%$ in the USA over the past decade, ${ }^{24,25}$ a growth surge driven by US births rather than by immigration. ${ }^{24}$ Unfortunately, the preterm birth rate among Hispanics has increased by $10 \%$ over the past decade, ${ }^{26}$ and this increase in prematurity has been only partly explained. Research has demonstrated that, in the Mexican-American population, the degree of acculturation involving the loss of traditional family and social support may affect family cohesion and have a direct effect on birth outcomes; Mexican-Americans who are more acculturated have higher PTB rates. ${ }^{27,28}$ For the purposes of this investigation, acculturation may be considered an ongoing process that occurs as immigrant groups gain increasing exposure to the beliefs, traits, identifications, and lifestyles of the dominant culture. This exposure and/ or transition to the dominant culture, versus maintenance of the heritage culture, may cause stress and overwhelming challenges for the women as they are also faced with the challenges of being pregnant. We therefore proposed that acculturation would increase the physiological stress response of cortisol and increase the risk of PTB. In addition, MexicanAmericans may encounter other particularly stressful life situations such as lower socioeconomic status, as well as difficulty accessing services and medical care. ${ }^{29}$ Because of the centrality of the family in the Mexican-American culture, we also proposed that family cohesion might be a protective factor to buffer some of the stress during pregnancy. ${ }^{30}$

Therefore, a second potential factor influencing stress may be family cohesion, defined as the feeling of connectedness between family members. ${ }^{31}$ Definitions of family cohesion usually also include affective characteristics of family relationships such as support, affect, and helpfulness. ${ }^{31-33}$ It is purported that levels of cohesion are an index of the interpersonal environment of the family and are associated with family effectiveness in dealing with developmental change and stress. ${ }^{34}$ Indeed, researchers examining family cohesion in a variety of populations have found that perception of increased family cohesion predicted overall emotional well-being and appeared to have a protective effect against emotional distress and mental and physical symptoms. ${ }^{35,36}$

There is, however, a paucity of research describing the mechanisms by which family cohesion and its interactions 
with the physiological stress response (eg, cortisol) may lead to birth outcomes, particularly in Mexican-American women at differing stages of acculturation. A cohesive family may be a protective factor for acculturating women that is associated with reduced stress, which can be measured by cortisol, a critical mediator of birth outcomes. ${ }^{37}$ As part of a larger study with multiple biomarkers of the endocrine and immune system that used a psychoneuroimmunological framework, ${ }^{38}$ the purpose of this analysis was to examine the effects of two psychosocial/ cultural factors (family cohesion and acculturation) as potential antecedents of the neuroendocrine stress response (cortisol) to predict PTB in a cohort of Mexican American women at midgestation. We hypothesized that:

1. Greater acculturation would predict higher cortisol levels and a higher risk of PTB.

2. Lower family cohesion scores would predict higher cortisol levels and a higher risk of PTB.

\section{Materials and methods Participants}

This cross-sectional study of PTB in Mexican-American women enrolled 470 women at 22-24 weeks gestation from multiple locations in Texas from 2003-2007. Approximately half the participants $(n=279,54 \%)$ were recruited from five community clinics in the Austin area. The remainder of the sample was recruited from a community clinic in the Houston area $(n=68,13 \%)$ and five private practice physicians' clinics in the San Antonio area $(n=172,33 \%)$. Women were included only if they had a singleton intrauterine pregnancy, selfidentified as Mexican-American ethnicity, and were able to read either Spanish or English. Convenience sampling was used to recruit all women who met the inclusion criteria. Women were excluded if they had any of the following conditions: cervical or uterine abnormalities, preeclampsia at the time of data collection (22-24 weeks gestation), congenital anomalies determined by ultrasound, placenta previa and/or cervicovaginal bleeding, blood group isoimmunization, heart and/or kidney disease, diabetes requiring medication, autoimmune disorders, asthma requiring medication, use of oral steroids within the last month, and diagnosis of a major mental disorder. Women with gestational diabetes were included only if they were diet-controlled at delivery. Participants who developed preeclampsia after data collection and prior to birth remained in the study. The study was approved by the Institutional Review Board at The University of Texas Health Science Center in San Antonio and the University of Texas Medical Branch in Galveston; all participants signed informed consent documents.

\section{Procedures}

The recruiter reviewed prenatal charts of possible participants before 22 weeks gestation and recruited participants before or after routine prenatal visits. The research nurse scheduled a separate visit for the data collection during the 22- to 24-week gestational period at which time the benefits and risks of the study were described and informed consent was obtained in either Spanish or English. Demographic data such as age, income, marital status, education, insurance, nativity (foreignborn versus US-born), gravidity, and parity, including history of PTB, were collected along with questionnaires on family cohesion and acculturation. Data collected from the prenatal record included (1) height and pre-pregnancy weight to calculate body mass index and (2) history of number and type of infections up until the point of data collection. A urine sample was collected for cotinine measurement and illicit drug testing and venous blood was obtained for cortisol measurement. Blood samples were obtained from a onetime venipuncture from 1-3 pm only, to control for diurnal variation in cortisol levels. Participants were encouraged to relax in a comfortable chair and were given the questionnaires first (30-minute period) to avoid increasing their acute stress response immediately prior to the blood draw. After delivery, maternal and infant outcomes were obtained from hospital medical records. Ultrasound less than 20 weeks was used as optimal dating criteria to establish gestational age at delivery. PTB was defined as parturition before 37 weeks 0 days gestational age and included both spontaneous PTB and medically indicated PTB due to preeclampsia.

\section{Measures}

\section{Family cohesion}

Family cohesion was assessed through a method developed by Vega et al in 1986, ${ }^{39}$ using the Family Adaptability and Cohesion Evaluation Scales (FACES)-II. ${ }^{40}$ This subscale of FACES consists of eight items addressing topics such as group problem solving, adherence to family decisions, family shared space and activity, feelings of family closeness, and enjoyment in family activities. Cronbach's $\alpha$ was 0.84 for the sample.

\section{Acculturation}

Acculturation was measured using a self-report instrument, the Language Proficiency subscale (LPS) of the Bidimensional Acculturation Scale (BAS) for Mexican-Americans. ${ }^{41}$ The scale has high internal consistency and high validity coefficients in Mexican-American and Central-American people. ${ }^{42}$ Psychometric analysis has shown that language 
items, compared with other constructs, explain most of the variance of acculturation scales. ${ }^{43}$ The LPS provides results for English proficiency (considered part of the receiving culture of USA), Spanish proficiency (considered more traditional or retaining the heritage of the Mexican-American culture), and bilingual participants (considered an integration of both cultures). In addition to the LPS of the BAS, years in the USA and country of birth were also used as measures of acculturation.

\section{Stress as measured by cortisol}

After obtaining the blood, samples were centrifuged at $2000 \mathrm{rpm}$ for 10 minutes. Shortly afterwards, the serum was aliquoted into polypropylene tubes and stored at $-80^{\circ} \mathrm{C}$. Cortisol was measured by enzyme-linked immunosorbent assay (ELISA) and read spectrophotometrically using a $\mu$-Quant R 141 reader (Bio-Tek Instruments, Inc, Winooski, VT, USA). Cortisol was assayed with a kit from Diagnostic Systems Laboratories (Webster, TX, USA). In order to control for potential differences in laboratory technique, the same laboratory technician conducted the analyses blinded to subject names and histories. The interassay coefficient of variation was less than $15 \%$.

\section{Statistical analysis}

The primary goal of our analysis was to examine the effects of family cohesion and acculturation to predict cortisol and PTB. Prior to constructing statistical models, we assessed seven potentially confounding variables to determine if they were sufficiently related to the outcome to be included in the final model. These were history of preterm birth, preeclampsia, and marital status, infections during pregnancy, maternal age, gravida, and gestation at data collection. We included only confounding variables that demonstrated a direct effect on the outcome due to the number of preterm births $(n=33)$ in the sample. There are no firm guidelines stating the number of covariates per event, but the heuristic guidelines presented by Hosmer and Lemeshow ${ }^{44}$ to avoid over-fitting the data, indicate that a reasonable ratio of covariates to events is ten cases for each covariate. Thus, we used the following sequence to assess the potentially confounding variables and interaction terms for inclusion in models: ${ }^{4}$ (1) Pearson's $\chi^{2}$ tests between each of the categorical independent variables and the outcome and $t$-tests using the outcome as an independent variable for each of the continuous variables were conducted to determine whether the variables were related to the outcome; (2) nonsignificant variables in step 1 were excluded from the model using a $P$-value of 0.20 to ensure that marginal effects are not excluded; (3) each remaining variable was assessed in a multivariable model; (4) categorical variables in the model were assessed for appropriate categories and linearity was assessed for continuous variables; and (5) interactions were fit. The potentially confounding variables that remained after this sequence, history of PTB and preeclampsia at delivery, remained in all subsequent logistic regression models. The statistical effects of family cohesion and acculturation were assessed by constructing interaction terms, which, if significant, indicate that the relationship between cortisol and PTB is moderated (ie, the relationship varies in strength depending on the value of the moderator) by family cohesion and acculturation. We then fit our final models in which family cohesion, cortisol, and the interaction between family cohesion and cortisol were regressed on PTB. In a second model, acculturation, cortisol, and the interaction between acculturation and cortisol were regressed on PTB. In the acculturation model, acculturation was a latent variable with three indicators (language proficiency, years in the USA, and country of birth). Cortisol was log-transformed to assure a normal distribution. Mplus statistical software (Muthén and Muthén version 7) was used to fit all logistic regression models described in this phase of the analysis. SAS (version 9.2; SAS Institute Inc, Cary, NC, USA) was used for $\chi^{2}$ tests and $t$-tests.

\section{Missing data}

We used multiple imputations to account for missing data using Mplus to generate 20 data sets in which the missing values were replaced using Bayesian analysis ${ }^{45}$ to generate plausible values for missing data. The imputed data sets were analyzed separately and results were combined to test the null hypothesis that model parameters were zero across the models using the 20 imputed data sets.

\section{Results}

\section{Sample characteristics}

Table 1 shows the characteristics of the sample. There were 33 PTBs in the sample, 28 spontaneous and five medically indicated due to preeclampsia. There were significant differences in the percentage of PTBs for participants with a history of PTB compared to those without such history and for participants who were born in the USA versus those who were foreign born. There was a significantly higher percentage of PTB for participants having a positive Group B strep culture prior to delivery versus those who had a negative culture. There were 33 total cases of preeclampsia 
Table I Sample characteristics

\begin{tabular}{|c|c|c|}
\hline & $\%$ total $(n)$ & $\%$ preterm (n) \\
\hline \multicolumn{3}{|l|}{ Demographics } \\
\hline \multicolumn{3}{|l|}{ Age $(n=470)$} \\
\hline $14-17$ years & $5.9(28)$ & $7.1(2)$ \\
\hline $18-25$ years & $54.9(258)$ & $7.4(19)$ \\
\hline $26-40$ years & $39.2(184)$ & $9.2(11)$ \\
\hline \multicolumn{3}{|l|}{ Household income, USD } \\
\hline \multicolumn{3}{|l|}{$(n=422)$} \\
\hline$<\$ 25,000$ & 71.1 (335) & $6.9(23)$ \\
\hline$\geq \$ 25,000$ & $17.7(83)$ & $8.4(7)$ \\
\hline \multicolumn{3}{|l|}{ Marital status $(n=469)$} \\
\hline Married & $42.4(198)$ & $7.6(15)$ \\
\hline Not married & $57.6(269)$ & $6.7(18)$ \\
\hline \multicolumn{3}{|l|}{ Country of birth $(n=470)$} \\
\hline US-born & $31.8(149)$ & $10.1(15)^{*}$ \\
\hline Foreign-born & $57.6(269)$ & $5.6(18)^{*}$ \\
\hline \multicolumn{3}{|l|}{ Education $(n=467)$} \\
\hline$<$ High school & $83.3(389)$ & $7.2(28)$ \\
\hline >High school & $16.7(78)$ & $6.4(5)$ \\
\hline \multicolumn{3}{|c|}{ Medical insurance $(n=466)$} \\
\hline Yes & 34.1 (159) & $10.1(16)$ \\
\hline No & $65.9(307)$ & $5.5(17)$ \\
\hline \multicolumn{3}{|l|}{ Other characteristics } \\
\hline \multicolumn{3}{|l|}{ Body mass index $(n=459)$} \\
\hline Underweight $<18.5$ & $6(28)$ & II.I (3) \\
\hline Normal I8.5-24.9 & $42.1(196)$ & $8.2(16)$ \\
\hline Overweight 25-29.9 & $29(135)$ & $6.7(9)$ \\
\hline Obese $>30$ & $23(107)$ & $4.7(5)$ \\
\hline \multicolumn{3}{|c|}{ Infection at delivery $(n=470)$} \\
\hline UTI & $3.6(17)$ & $5.9(1)$ \\
\hline No UTI & $96.4(453)$ & $6.8(32)$ \\
\hline GBS $(n=430)$ & $16.7(72)$ & $12.5(9)^{*}$ \\
\hline No GBS & $83.3(358)$ & $4.8(17)$ \\
\hline STI $(n=434)$ & $3.5(15)$ & $0(0)$ \\
\hline No STI & $96.5(419)$ & $6.7(28)$ \\
\hline \multicolumn{3}{|l|}{ PTB history $(n=47 I)$} \\
\hline Yes & $7.9(37)$ & $16.2(6)^{*}$ \\
\hline No & $92.1(434)$ & $6.2(27)$ \\
\hline \multicolumn{3}{|l|}{ Preeclampsia at delivery } \\
\hline \multicolumn{3}{|l|}{$(n=433)$} \\
\hline Yes & $7.6(33)$ & $15.2(5)^{*}$ \\
\hline No & $92.4(400)$ & $6.0(24)$ \\
\hline \multicolumn{3}{|l|}{ Gravida $(n=463)$} \\
\hline 1 & $27.7(128)$ & $8.6(\mathrm{II})$ \\
\hline 2 & $30.2(140)$ & $3.6(5)$ \\
\hline 3 & $22.9(106)$ & $9.4(10)$ \\
\hline 4 & $11.0(5 \mathrm{I})$ & $7.8(4)$ \\
\hline 5 & $4.3(20)$ & $0.0(0)$ \\
\hline $6+$ & $3.9(18)$ & $16.7(3)$ \\
\hline
\end{tabular}

Notes: Percentages and frequencies represent the number of non-missing cases for each of the sample characteristics. $* P<0.05$.

Abbreviations: GBS, group B Streptococcus; PTB, preterm birth; STI, sexually transmitted infection; UTI, urinary tract infection.

at delivery (five PTBs and 28 term deliveries). The data from these participants were included in the analysis as their stress (cortisol) levels did not significantly differ from those participants without preeclampsia. Eighteen participants were diagnosed with gestational diabetes prior to delivery but after data collection; the data from these participants were also included in the analysis as their cortisol did not differ significantly from those who were not diabetic. Except for one mother using an opiate who delivered a term low-birthweight infant, no other preterm or low-birth-weight infants had mothers who used illicit drugs, determined by urine testing. In addition, the number of smokers in the total sample was very low $(n=3)$ and smoking was thus not used as a covariate.

The 33 PTBs included 15 births at 36 weeks, nine at 35 weeks, six at 33 weeks, one at 32 weeks, one at 31 weeks, and one at 23 weeks. The percentage of PTB in this sample was low (7\%) compared to the national average of $12 \%$ for Mexican-Americans. A possible explanation for this low percentage is that a large number of participants were primarily proficient in Spanish $(n=290)$ or born in Mexico $(n=320)$, and were new immigrants in the USA. These participants had a $5 \%$ PTB rate, in contrast to the $10 \%$ rate of the women who were proficient in English $(n=178)$ or were born in the USA $(n=149)$. Thus, as predicted by the Hispanic paradox that states that, despite worse conditions, Mexican-American immigrants have healthier birth outcomes than Mexican-Americans who are born in the USA, the majority of the women (new immigrants) in our study had healthier birth outcomes, thus lowering the percentage of PTBs.

\section{Comparisons of acculturation levels and family cohesion}

Independent $t$-tests were conducted to determine differences in family cohesion by acculturation using language proficiency on the LPS. The mean of the family cohesion scale was 26.9 for the participants who had high English proficiency $(n=67)$ versus a mean of 29.8 for the participants who had higher Spanish proficiency $(\mathrm{n}=275)(t=-4.05, P<0.001$ [confidence interval (CI) $-4.32 ;-1.4])$. The mean of family cohesion was 30.5 for participants who scored in the bilingual category $(\mathrm{n}=120 ; t=-3.73, P<0.001[\mathrm{CI}-5.5 ;-1.7])$.

\section{Comparisons of acculturation levels and cortisol levels}

Independent $t$-tests were conducted to determine differences in cortisol levels by acculturation using language proficiency on the LPS. The mean of cortisol was $11.6 \mathrm{ng} / \mathrm{mL}$ for participants who had high English proficiency versus $18.9 \mathrm{ng} / \mathrm{mL}$ for participants who had high Spanish proficiency $(\mathrm{n}=274)$ $(t=-6.98 ; P<0.001$ [confidence interval (CI)1.05; -9.46]). The mean of cortisol was $14.06 \mathrm{ng} / \mathrm{mL}$ for participants who scored in the bilingual category $(\mathrm{n}=119)(t=-2.05 ; P=0.04$ [CI $1.19 ;-4.82])$. 


\section{Model building}

\section{Steps in model building}

In the first step in the five-step process we examined the relationship between each independent variable and the dependent variable. Two variables exhibited significant bivariate relationships with preterm birth - history of preterm birth $\left(\chi^{2}=5.20, P=0.02\right)$ and preeclampsia $\left(\chi^{2}=4.09\right.$, $P=0.04)$ - and were thus retained in the second step. In the third step, history of preterm birth (estimate/standard error $[\mathrm{E} / \mathrm{SE}]=2.26, P=0.024)$ and preeclampsia $(\mathrm{E} / \mathrm{SE}=1.96$, $P=0.050)$ remained significant in a multiple variable model. In the fourth step, the linearity of the continuous variables (cortisol, family cohesion, and acculturation) was assessed; there were no significant non-linear relationships observed. In the fifth step, all combinations of cortisol, family cohesion, and acculturation were separately examined with the two potentially confounding variables, history of PTB and preeclampsia. The interaction between family cohesion and history of PTB (E/SE $-2.12, P=0.034)$ was the only significant interaction and was thus retained in models containing family cohesion.

In analyzing the interaction between cortisol and family cohesion, we estimated the odds ratio for cortisol at 1 standard deviation below the mean of family cohesion was $2.20(P=0.017)$ and $0.69(P=0.110)$ at 1 standard deviation above the mean. The odds ratio for family cohesion at 1 standard deviation below the mean of cortisol was $2.28(P=0.026)$ and $0.71(P=0.190)$ at 1 standard deviation above the mean. That is, participants with lower family cohesion were more likely to have higher cortisol levels. In Table 2, we show that preeclampsia and the interactions between history of PTB and family cohesion, and cortisol and family cohesion are significant. These interactions are seen in Figures 1 and 2, respectively. In Figure 1, we see the paradoxical relationship between family cohesion, cortisol, and PTB; when family cohesion

Table 2 Parameter estimates for family cohesion moderator model

\begin{tabular}{lllll}
\hline Model parameter & Estimate & $\begin{array}{l}\text { Standard } \\
\text { error }\end{array}$ & $\begin{array}{l}\text { Estimatel } \\
\text { standard } \\
\text { error }\end{array}$ & $P$ \\
\hline Intercept & -2.97 & 0.24 & 12.46 & 0.000 \\
Preeclampsia & 1.09 & 0.56 & 1.96 & 0.050 \\
History of PTB & 0.78 & 0.65 & 1.04 & 0.297 \\
Stress (log cortisol) & 0.20 & 0.18 & 1.16 & 0.247 \\
Family cohesion & 0.24 & 0.23 & 1.07 & 0.285 \\
History of PTB X family & -1.23 & 0.58 & -2.12 & 0.034 \\
cohesion & & & & \\
Stress X family cohesion & -0.58 & 0.23 & -2.57 & 0.010 \\
\hline
\end{tabular}

Abbreviation: PTB, preterm birth.

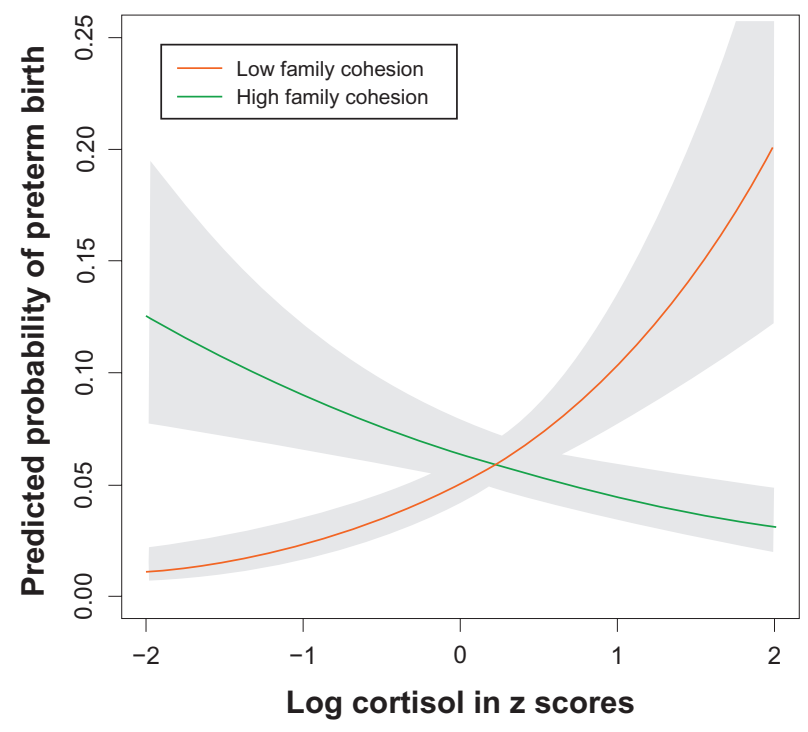

Figure I Family cohesion and cortisol predicting preterm birth. Grey areas represent standard errors. White areas represent regions of significant differences.

is high and cortisol is low and when family cohesion is low and cortisol is high, the probability of PTB increases. In Figure 2, we see a more direct relationship; when family cohesion is high, it acts to decrease the risk of PTB when there is a prior history of PTB, though this effect was detected during routine model-building steps and should thus be interpreted with caution.

In analyzing the interaction between cortisol and acculturation, we found that the odds ratio for cortisol at 1 standard deviation below the mean of acculturation was

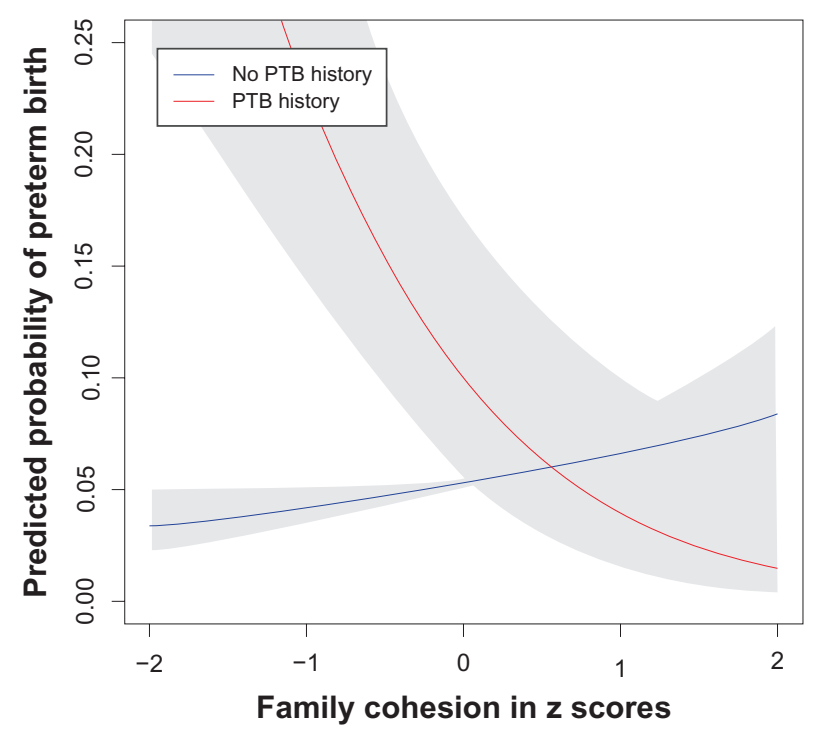

Figure 2 Family cohesion predicting preterm birth. Grey areas represent standard errors. White areas represent regions of significant differences. Abbreviation: PTB, preterm birth. 
$0.83(P=0.311)$ and $2.12(P=0.019)$ at 1 standard deviation above the mean and that the odds ratio for acculturation at 1 standard deviation below the mean of cortisol was $1.05(P=0.852)$ and $2.66(P=0.002)$ at 1 standard deviation above the mean. That is, more acculturated participants were more likely to have higher stress responses as measured by cortisol. In Table 3, we provide a model of PTB predicted by acculturation where preeclampsia, history of PTB, acculturation, and the interaction between cortisol and acculturation are significant. The interaction effect of acculturation and cortisol on PTB is seen in Figure 3, where the probability of PTB rises with increasing acculturation and increasing cortisol levels.

Because the final model contained more than the number of recommended terms given the rate of PTB in the sample, we confirmed the results of our primary findings using only the interactions and the terms that comprise the interactions as seen in Table 4. The cortisol by family cohesion interaction, the family cohesion by history of PTB interaction, and the acculturation by cortisol interaction remained significant in the smaller model.

\section{Discussion}

The primary purpose of this analysis was to examine the effects of two psychosocial/cultural factors (family cohesion and acculturation) as potential antecedents of the neuroendocrine stress response (cortisol) to predict PTB in a cohort of Mexican-American women at midgestation. We hypothesized that greater acculturation would predict higher cortisol levels and a higher risk of PTB. This hypothesis was supported. Acculturation was a direct predictor of PTB and predicted the rise in cortisol, which then predicted PTB (see Figure 3). Thus, acculturation may directly affect birth outcomes through the physiologic stress response involving a rise in cortisol resulting in negative birth outcomes, specifically PTB.

The second hypothesis was that lower family cohesion scores would predict higher cortisol levels and a higher

Table 3 Parameter estimates for acculturation moderator model

\begin{tabular}{lllll}
\hline Model parameter & Estimate & $\begin{array}{l}\text { Standard } \\
\text { error }\end{array}$ & $\begin{array}{l}\text { Estimate/ } \\
\text { standard } \\
\text { error }\end{array}$ & $\boldsymbol{P}$ \\
\hline Intercept & -2.89 & 0.23 & 12.49 & 0.000 \\
Preeclampsia & 1.17 & 0.56 & 2.09 & 0.037 \\
History of PTB & 1.22 & 0.54 & 2.26 & 0.024 \\
Stress (log cortisol) & 0.28 & 0.18 & 1.62 & 0.104 \\
Acculturation & 0.56 & 0.22 & 2.55 & 0.011 \\
Stress X acculturation & 0.51 & 0.21 & 2.43 & 0.015 \\
\hline
\end{tabular}

Abbreviation: PTB, preterm birth.

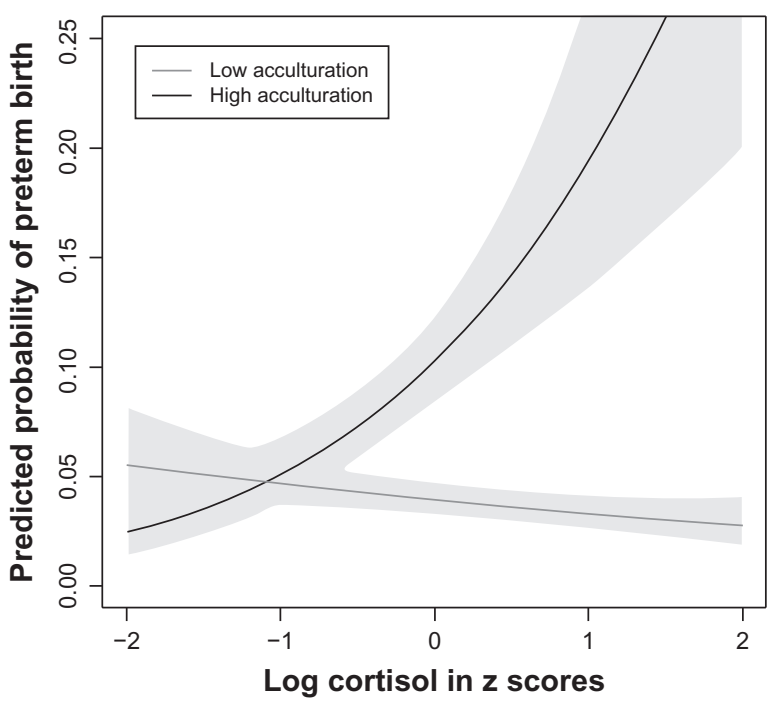

Figure 3 Acculturation and Cortisol Predicting Preterm Birth. Grey areas represent standard errors. White areas represent regions of significant differences.

risk of PTB. This hypothesis was partially supported. Lower levels of family cohesion and rising cortisol levels predicted an increased risk of PTB. However, there was an unanticipated effect of high family cohesion levels predicting lower cortisol levels, which were also associated with an increased risk of PTB (see Figure 1). These results are consistent with the idea that the HPA axis functions to establish equilibrium, or homeostasis, in the face of adverse stimuli, or stressors. ${ }^{12}$ Appropriate responsiveness of the HPA axis is essential for a sense of well-being and positive social interactions. The results of our analysis suggest that an imbalance in family functioning (either too much or too little) may be associated with disequilibrium in the HPA axis, with resultant changes in cortisol that may affect pregnancy outcomes. In other words, too much family cohesion and possible family enmeshment or too little family cohesion may lead to a state of chronic stress, resulting in HPA axis dysregulation and subsequent PTB.

In addition to the hypothesized predictive effects of acculturation and family cohesion, a routine assessment of potentially confounding variables revealed that pregnant women with a history of PTB had a higher risk of repeat PTB if they had lower family cohesion.

An important implication of the results of this study is that assessment of family functioning may be needed as part of a prenatal risk profile for PTB in Mexican-American women. Such assessments have been previously suggested to identify different subgroups of risk for MexicanAmerican women. ${ }^{36}$ Our results, in part, replicated previous results ${ }^{46}$ indicating that higher family cohesion may be 
Table 4 Supplemental parameter estimates for interaction models of family cohesion, acculturation and stress

\begin{tabular}{llllll}
\hline Model & Model parameter & Estimate & $\begin{array}{l}\text { Standard } \\
\text { error }\end{array}$ & $\begin{array}{l}\text { Estimate/standard } \\
\text { error }\end{array}$ \\
\hline Family cohesion & Intercept & -2.69 & 0.20 & 13.72 & $\begin{array}{l}\boldsymbol{P} \\
\end{array}$ \\
& Stress (log cortisol) & 0.20 & 0.17 & 1.14 & 0.255 \\
& Family cohesion & 0.02 & 0.20 & 0.08 & 0.936 \\
& Stress X family cohesion & -0.52 & 0.21 & -2.46 & 0.014 \\
Acculturation & Intercept & -2.61 & 0.20 & 13.35 & $<0.001$ \\
& Stress (log cortisol) & 0.31 & 0.17 & 1.87 & 0.061 \\
& Acculturation & 0.51 & 0.21 & 2.43 & 0.015 \\
& Stress X acculturation & 0.48 & 0.19 & 2.56 & 0.011 \\
\hline
\end{tabular}

protective for health. Our results supplement this previous work, but our findings suggest that family cohesion is protective only if the stress response is not chronically elicited resulting in an HPA dysregulation of cortisol. In such circumstances higher family cohesion may lead to poorer coping, chronic stress, and lower cortisol levels. Other studies have indicated that erosion of family cohesion or family bonding occurs with greater time in the USA. ${ }^{47}$ The findings of Cook et $\mathrm{al}^{48}$ implied that interventions to prevent family erosion during the integration process of acculturation are critical, especially for the mental health of Mexican-Americans. Our findings of low family cohesion, increasing levels of stress (cortisol), and increased risk of PTB also indicate the importance of assessment of family cohesion. Measures to assist families to decrease stress and work together during the developmental period of pregnancy and becoming parents may be vital. Referrals to social workers and mental health professionals may be indicated to assist with needed improvement in family functioning.

Another important implication of our findings is that this evidence adds to the body of knowledge of how acculturation predicts PTB. Our results confirmed those of D'Anna-Hernandez et al, ${ }^{49}$ which showed that acculturation predicted cortisol and, as such, is a risk factor for poor pregnancy outcomes, in this case PTB. Previous work ${ }^{27}$ has indicated the risk of acculturation in relationship to the reproductive hormones of progesterone and estriol, which are biochemically related to cortisol. Cortisol and acculturation levels may be important components of a risk profile to predict birth outcomes in Mexican-American women, a vulnerable population. By recognizing the interactions of psychosocial and cultural factors with biological markers of stress, health care providers can ascertain risks for PTB in Mexican-American Hispanics. Further research is needed to identify and test possible interventions to decrease the stress response and improve the protective ability of the family, or to teach the mother how to interact more effectively with her family. It may also be important to consider the timing of these results as they were obtained midpregnancy, and cortisol may not predict as well earlier in pregnancy. Therefore, these results may need to be replicated earlier in pregnancy to determine predictive accuracy.

This study had several notable strengths. First, the sample size was large enough to be able to detect variances in cortisol levels and acculturation levels. The large sample also facilitated ability to detect different levels of family cohesion. Another strength was the biomarker measurement of stress, adding physiological results to psychological and cultural measures. Use of a latent variable of acculturation (country of birth, years in the USA, and language proficiency) added strength as well, providing additional dimensionality to the concept of acculturation.

The study also had limitations. A principal limitation was the use of a one-time cortisol measurement. This study was part of a larger study that had multiple measures, including urine, vaginal, and blood samples. Because of the many biological samples being obtained, we decided to reduce participant burden by only collecting blood for serum cortisol measurement and to control for time of day in the afternoon when the levels naturally are lower than with the awakening response. Because of these methods, there was neither the ability to measure diurnal slope, as may be done with several measures when salivary cortisol is used, nor was there the ability to measure the cortisol awakening response. Another limitation to the study was the cross-sectional study design, which limits the ability to detect true causal relationships. The sample was a convenience sample, and as such, may not be truly representative of the population. Finally, the small number of PTBs (33) in the sample limited the number of independent variables that could be used in developing the prediction models.

Despite these limitations, the results from this study are important and have potential major public health implications. 
This research adds to the understanding of factors that predict cortisol and HPA dysregulation which has been implicated consistently as part of the pathogenesis of PTB. Future research might focus on a risk profile to refine and identify, by family functioning and degree of acculturation, those most at risk for PTB, and to identify novel therapies to reduce the physiological stress response of cortisol.

\section{Acknowledgment}

This study was funded by National Institutes of Health, National Institute of Nursing Research R01NR007891.

\section{Disclosure}

The authors report no conflicts of interest in this work.

\section{References}

1. Williamson DM, Abe K, Bean C, Ferré C, Henderson Z, Lackritz E. Current research in preterm birth. J Womens Health (Larchmt). 2008;17(10):1545-1549.

2. Behrman RE, Butler AS, editors. Preterm Birth: Causes, Consequences, and Prevention. Washington DC: National Academies Press; 2007.

3. Iams JD, Berghella V. Care for women with prior preterm birth. Am J Obstet Gynecol. 2010;203:89-100.

4. Wadhwa PD, Entringer S, Buss C, Lu MC. The contribution of maternal stress to preterm birth: issues and considerations. Clin Perinatol. 2011;38:351-384.

5. Paarlberg KM, Vingerhoets AJ, Passchier J, Dekker GA, Van Geijn HP. Psychosocial factors and pregnancy outcomes: a review with emphasis on methodological issues. J Psychosom Res. 1995;39:563-595.

6. Dunkel Schetter C. Psychological science on pregnancy: stress processes, biopsychosocial models, and emerging research issues. Annu Rev Psychol. 2011;62:531-558.

7. Ruiz RJ, Fullerton J, Brown C, Dudley DJ. Predicting risk of preterm birth: the roles of stress, clinical risk factors, and corticotrophin-releasing hormone. Biol Res Nurs. 2002;4:54-64.

8. Ruiz RJ, Fullerton J, Dudley DJ. The interrelationship of maternal stress, endocrine factors and inflammation on gestational length. Obstet Gynecol Surv. 2003;58(6):415-428.

9. Hobel CJ, Goldstein A, Barrett ES. Psychosocial stress and pregnancy outcome. Clin Obstet Gynecol. 2008;51:333-348.

10. Lockwood CJ. Recent advances in elucidating the pathogenesis of preterm delivery, the detection of patients at risk, and preventative therapies. Curr Opin Obstet Gynecol. 1994;6(1):7-18.

11. Wadhwa PD, Culhane JF, Rauh V, et al. Stress, infection and preterm birth: a biobehavioural perspective. Paediatr Perinat Epidemiol. 2001; 15 Suppl 2:17-29.

12. Vrekoussis T, Kalantaridou SN, Mastorakos G, et al. The role of stress in female reproduction and pregnancy: an update. Ann NY Acad Sci. 2010;1205:69-75.

13. Diego MA, Jones NA, Field T, et al. Maternal psychological distress, prenatal cortisol, and fetal weight. Psychosom Med. 2006;68:747-753.

14. Kalra S, Einarson A, Karaskov T, Van Uum S, Koren G. The relationship between stress and hair cortisol in healthy pregnant women. Clin Invest Med. 2007;30:E103-E107.

15. Obel C, Hedegaard M, Henriksen TB, Secher NJ, Olsen J, Levine S. Stress and salivary cortisol during pregnancy. Psychoneuroendocrinology. 2005;30:647-656.

16. Erickson K, Thorsen P, Chrousos G, et al. Preterm birth: associated neuroendocrine, medical, and behavioral risk factors. J Clin Endocrinol Metab. 2001;86:2544-2552.
17. Field T, Hernandez-Reif M, Diego M, Figueiredo B, Schansberg S, Kuhn C. Prenatal cortisol, prematurity and low birthweight. Infant Behav Dev. 2006;29:268-275.

18. Phocas I, Sarandakous A, Rizos D. Maternal serum total cortisol levels in normal and pathologic pregnancies. Int J Gynaecol Obstet. 1990;31:3-8.

19. Buss C, Entringer S, Reyes JF, et al. The maternal cortisol awakening response in human pregnancy is associated with length of gestation. Am J Obstet Gynecol. 2009;201:398. e1-e8.

20. Entringer S, Buss C, Andersen J, Chicz-DeMet A, Wadhwa PD. Ecological momentary assessment of maternal cortisol profiles over a multiple day period predict the length of human gestation. Psychosom Med. 2011;73:469-474.

21. Cohen S, Doyle WJ, Baum A. Socioeconomic status is associated with stress hormones. Psychosom Med. 2006;68:414-420.

22. Suglia SF, Cohen S, Rich-Edwards J, Staudenmayer J, Enlow MB, Wright RJ. Cumulative stress and cortisol disruption among black and Hispanic pregnant women in an urban cohort. Psychol Trauma. 2010;2: 326-334.

23. Glynn LM, Schetter CD, Chicz-DeMet A, Hobel CJ, Sandman CA. Ethnic differences in adrenocorticotropic hormone, cortisol, and corticotrophin releasing hormone during pregnancy. Peptides. 2007;28: $1155-1161$

24. Ceasar S. Hispanic population tops 50 million in US Los Angeles Times. March 24, 2011. Available from: http://articles.latimes.com/2011/mar/24/ nation/la-na-census-hispanic-20110325. Accessed March 10, 2012.

25. Mexican American population of the United States, 2012 [webpage on the Internet]. Available from: http://www.census.gov/compendia/statab/ cats/population. Accessed March 10, 2012.

26. MacDorman M. Race and ethnic disparities in fetal mortality, preterm birth, and infant mortality in the United States: an overview. Semin Perinatol. 2011;35:200-208.

27. Ruiz RJ, Saade GR, Brown CE, et al. The effect of acculturation on progesterone/estriol ratios and preterm birth in Hispanics. Obstet Gynecol. 2008;111(2 Pt 1):309-316.

28. Flores ME, Simonsen SE, Manuck TA, Dyer JM, Turok DK. The "Latina epidemiologic paradox": contrasting patterns of adverse birth outcomes in US-born and foreign-born Latinas. Womens Health Issues. 2012;22(5):e501-e507.

29. Farley T, Galves A, Dickinson LM, Perez Mde J. Stress, coping, and health: a comparison of Mexican immigrants, Mexican-Americans, and non-Hispanic whites. J Immigr Health. 2005;7:213-219.

30. Leidy MS, Guerra NG, Toro RI. Positive parenting, family cohesion, and child social competence among immigrant Latino families. J Fam Psychol. 2010;24:252-260.

31. Olson DH, McCubin H, Barnes H, Larsen A, Muxem A, Wilson N. Families: What Makes Them Work? Beverly Hills CA: Sage Publishing, Inc; 1983.

32. Baer J. Is family cohesion a risk or protective factor during adolescent development? J Marriage Fam. 2002;64:668-675.

33. North RJ, Holahan CJ, Moos RH, Cronkite RC. Family support, family income, and happiness: a 10 year perspective. J Fam Psychol. 2008;22: 475-483.

34. Baer J. The effects of family structure and SES on family processes in early adolescence. J Adolesc. 1999;22:341-354.

35. Weisman A, Rosales G, Kymanlainen J, Armesto J. Ethnicity, family cohesion, religiosity and general emotional distress in patients with schizophrenia and their relatives. $J$ Nerv Ment Dis. 2005;193: 359-368.

36. Balcazar H, Krull JL, Peterson G. Acculturation and family functioning are related to health risks among pregnant Mexican American women. Behav Med. 2001;27:62-70.

37. Wadhwa PD, Dunkel-Schetter C, Chicz-DeMet A, Porto M, Sandman CA. Prenatal psychosocial factors and the neuroendocrine axis in human pregnancy. Psychosom Med. 1996;58:432-446.

38. Ader R. Psychoneuroimmunology, 1st ed. Orlando, FL: Academic Press; 1981. 
39. Vega W, Patterson T, Sallis J, Nader P, Atkins C, Abramson I. Cohesion and adaptability in Mexican Americans and Anglo families. J Marriage Fam. 1986;48:857-867.

40. Olson DH, Russell CS, Sprenkle DH. Circumplex model of marital and family systems: VI. Theoretical update. Fam Process. 1983;22: 69-83.

41. Marin G, Gamba RJ. A new measurement of acculturation for Hispanics: the Bidimensional Acculturation Scale for Hispanics (BAS). Hisp $J$ Behav Sci. 1996;18:297-316.

42. Marin G. Issues in the measurement of acculturation among Hispanics. In: Geisinger KF, editor. Psychological Testing of Hispanics. Washington, DC: American Psychological Association; 1992:23-51.

43. Cabassa LJ. Measuring acculturation: where we are and where we need to go. Hisp J Behav Sci. 2003;25:127-146.

44. Hosmer DW, Lemeshow S. Applied Logistic Regression, 2nd ed. New York, NY: John Wiley and Sons; 2000.
45. Graham JW. Missing data analysis: making it work in the real world. Annuv Rev Psychol. 2009;60:549-576.

46. Sherraden M, Barrera R. Poverty, family support, and well-being of infants: Mexican immigrant women and childbearing. J Sociol Soc Welf. 1996;23:27-54

47. Hovey JD, King CA. Acculturation stress, depression and suicidal ideation among immigrant and second-generation Latino adolescents. J Am Acad Child Adolesc Psychiatry. 1996;35:1183-1192.

48. Cook B, Alegria M, Lin JY, Guo J. Pathways and correlates connecting Latinos' mental health with exposure to the United States. Am J Public Health. 2009;99:2247-2254.

49. D'Anna-Hernandez KL, Hoffman MC, Zerbe GO, Coussons-Read M, Ross RG, Laudenslager ML. Acculturation, maternal cortisol, and birth outcomes in women of Mexican descent. Psychosom Med. 2012;74: 296-304.
International Journal of Women's Health

\section{Publish your work in this journal}

The International Journal of Women's Health is an international, peerreviewed open-access journal publishing original research, reports, editorials, reviews and commentaries on all aspects of women's healthcare including gynecology, obstetrics, and breast cancer. The manuscript management system is completely online and includes

\section{Dovepress}

a very quick and fair peer-review system, which is all easy to use. Visit http://www.dovepress.com/testimonials.php to read real quotes from published authors.

Submit your manuscript here: http://www.dovepress.com/international-journal-of-womens-health-journal 